\title{
Comparativo de las condiciones de transporte terrestre de carga entre los países miembro de la Alianza del Pacífico
}

\author{
Comparative study of the conditions of transportation of cargo between \\ the member countries of the Alliance of the Pacific
}

Recibido: 15-11-2015 • Aprobado: 28-06-2016 • Página inicial: 155 - Página final: 181

\author{
Jose Jaime Baena Rojas* \\ Diego Alejandro Castaño Villa** \\ María Alejandra Tabares Castrillón***
}

Resumen: este artículo analiza aspectos generales estratégicos asociados a las condiciones de transporte terrestre dentro de los países de la Alianza del Pacífico. En este sentido, son comparados factores infraestructura de transporte, de inversión y de competitividad; en cuyo caso son considerados asimismo una multiplicidad de variables que pueden definir en cierto modo el nivel de preparación y de capacidad de cada país para atender el tránsito terrestre de mercancías. Tras la aplicación de un método de normalización para cada variable además de hacer uniformes los datos en una escala de uno a cinco; los resultados obtenidos sugieren que México es el país con mayor nivel de preparación dadas sus actuales condiciones de transporte terrestre.

Palabras clave: integración económica, competitividad, transporte, infraestructura, inversión.
Abstract: This article discusses general aspects associated with the strategic conditions for inland transport within the countries of the Pacific Alliance. In this regard, are compared factors transport infrastructure, investment and competitiveness; in which case are considered also a multiplicity of variables that can define in some way the level of preparedness and capacity of each country to meet the land transit of goods. After the implementation of a method of standardization for each variable in addition to make uniform data on a scale of one to five; the results obtained suggest that Mexico is the country with the highest level of preparation given their current conditions of land transport.

Keywords: Economic integration, competitiveness, transport, infrastructure, investment.

$$
\text { JEL: F02, H54, L1, R40 }
$$

* Ph.D. en Derecho y Ciencia Política, MSc. en Internacionalización, Especialista en Comercio Internacional, Politólogo y Profesional en Negocios Internacionales. Docente Investigador Universidad de Medellín. jjbaena@udem.edu.co

** Tecnólogo en Comercio Exterior, Tecnológico de Antioquia Institución Universitaria, Medellín Colombia.diego-1794@hotmail.com

***Tecnóloga en Comercio Exterior, Tecnológico de Antioquia Institución Universitaria, Medellín Colombia. alejita115t@hotmail.com 


\section{Conditions comparatifs de transport terrestre de marchandises entre les pays membres de I'Alliance du Pacifique}

Résumé: cet article analyse aspects généraux stratégiques associés à des conditions de transport terrestre dans les pays de l'Alliance du Pacifique. À cet égard, sont comparées facteurs infrastructures de transport, d'investissement et de compétitivité; dans ce cas sont considérés comme également une multitude de variables peuvent être définies dans une certaine mesure le niveau de préparation et de capacité de chaque pays pour répondre le transit terrestre de marchandises. Après la mise en oeuvre d'une méthode de normalisation pour chaque variable en outre de faire des uniformes des données à une échelle de un à cinq; les résultats obtenus indiquent que le Mexique est le pays avec plus de préparation en raison de leurs conditions de transport terrestre.

Mots-clés: intégration économique, compétitivité, transports, infrastructure, investissement.

\section{Estudo comparativo das condições de transporte de carga entre os países membros da Aliança do Pacífico}

Resumo: este artigo discute aspectos gerais associadas com as condições estratégicas para o transporte terrestre nos países da Aliança do Pacífico. A este respeito, são comparados factores infra-estruturas de transportes, o investimento e a competitividade; caso em que são considerados também uma multiplicidade de variáveis que podem definir de alguma forma o nível de preparação e a capacidade de cada país para atender o trânsito terrestre de mercadorias. Após a aplicação de um método de padronização para cada variável além de uniformizar os dados em uma escala de um a cinco; os resultados obtidos sugerem que o México é o país com o maior nível de preparação tendo em conta as suas actuais condições de transporte terrestre.

Palavras-chave: a integração económica, competitividade, transportes, infraestruturas, investimentos. 


\section{Introducción}

Se toma como punto de partida la premisa de que la infraestructura es uno de los requerimientos básicos y prioritarios para el desarrollo de una región, pues la adecuación y eficiencia de la misma articulada con su territorio permite a la región obtener un posicionamiento a nivel mundial debido a que los niveles de competitividad aumentan y la calidad de vida de sus habitantes mejora. Por tal razón la adecuación de la infraestructura se ha convertido en un reto para las regiones el cual demanda considerables inversiones, avanzada tecnología, una gran capacidad de gestión y un marco institucional eficiente (Vassallo y Izquierdo, 2010).

Las regiones se han convertido en redes económicas gracias a los procesos de integración, donde la competitividad se desarrolla con facilidad gracias al libre acceso y a los programas de integración que se llevan entre los países, los cuales pretenden ejecutar procesos de inversión en infraestructura para así generar competitividad en las regiones más rezagadas en este aspecto (García, 2007).

Considerando lo anterior se destaca la pertinencia que poseen las condiciones de transporte para el progreso de un país o una región, en este caso se tomara como referencia el transporte terrestre, el cual utiliza las carreteras como medio para la movilidad de carga y pasajeros desde un punto de origen hasta uno de destino. Una carretera debe adaptarse a los requerimientos que demandan los usuarios del servicio, marchar con los tiempos, y así garantizar el desplazamiento de carga y pasajeros en óptimas condiciones de rapidez, comodidad y seguridad, lo que implica el desarrollo de nuevas vías y modernización de las ya existentes (Papí, 2012).

Tomando como eje central la relevancia de las condiciones de transporte terrestre en el desarrollo de un país o región, el presente artículo pretende determinar cuán competitivo es el transporte terrestre para la movilización de flujos comerciales en Chile, Colombia, México y Perú; todos estos miembros de la Alianza del Pacífico y cuál es la influencia que tiene el sector público y privado para responder a los retos en materia de transporte por carretera. En definitiva, la infraestructura tiene impactos sobre las economías, pues si no se realiza el aprovisionamiento y adecuación de la misma en términos de calidad y cantidad; el crecimiento económico y la distribución de los ingresos se verán afectados, por otra parte en el caso de la infraestructura vial; la inadecuada provisión genera costos excesivos para los usuarios, lo que limita la prestación del servicio de transporte (Urrunaga Pascó-Font, 2009). 


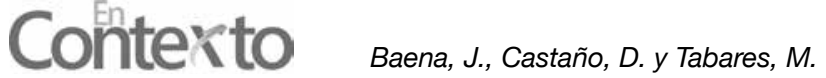

En consecuencia, el presente artículo posee un enfoque cuantitativo en cual con datos secundarios proporcionados por instituciones a nivel internacional; se pretende caracterizar y posteriormente comparar el entorno de las condiciones del transporte terrestre de los cuatro países miembros de la Alianza del Pacífico; profundizando en tres categorizaciones: infraestructura de transporte terrestre, inversión y competitividad, las cuales permitan determinar la posición de cada uno de estos países en lo que respecta a las condiciones para movilizar carga por carretera y de la misma manera conocer cuáles son los retos que tiene cada país para obtener una mayor competitividad a nivel internacional.

La pertinencia de esta investigación con los negocios internacionales se encuentra fundamentada en la competitividad generada por las adecuadas condiciones de transporte terrestre en países y regiones, esto debido inicialmente a la optimización de costos y tiempos asociados al transporte de flujos comerciales dentro de su espacio físico. Esta investigación brinda a los sectores público y privado la opción de conocer la situación de las condiciones de transporte y el impacto que estas tienen sobre las operaciones de comercio internacional de mercaderías, para ambos sectores permitirá establecer planes de acción en función de la competitividad. Igualmente cabe indicar que la presente investigación se realizó con el fin de afianzar y plasmar los conocimientos adquiridos durante el desarrollo del pregrado en negocios internacionales.

Finalmente dentro del artículo se establecen conclusiones en torno a las condiciones del transporte terrestre de carga de los cuatro países miembros de la Alianza del Pacífico, considerando el estado en el cual se encuentra su infraestructura en términos logísticos de cantidad y calidad, la inversión realizada en proyectos de transporte, y la situación competitiva de cada país en el contexto internacional.

\section{Metodología}

La investigación tiene un enfoque cuantitativo en el cual se recurre a fuentes secundarias que permitan obtener cifras para realizar un análisis y comparación de las mismas. Inicialmente se definen las variables inherentes a la temática de la investigación y se clasifican en categorías, las cuales corresponden a los temas mencionados en el marco teórico infraestructura de transporte terrestre, inversión y competitividad. De la misma manera se define la unidad de referencia de cada variable, tal como se puede observar en la Tabla 1. 
Tabla 1

Variables evaluadas para establecer comparativo entre los países miembros de la Alianza del Pacifico

\begin{tabular}{|c|c|c|c|c|}
\hline CATEGORIA & \multicolumn{4}{|c|}{ VARIABLES } \\
\hline $\begin{array}{c}\text { INFRAESTRUCTURA } \\
\text { DE TRANSPORTE } \\
\text { TERRESTRE }\end{array}$ & $\begin{array}{c}\text { Densidad vial } \\
\left(\mathrm{Km} / \mathrm{Km}^{2}\right)\end{array}$ & $\begin{array}{l}\text { Desempeño } \\
\text { logístico } \\
\text { (Puntaje) }\end{array}$ & $\begin{array}{l}\text { Calidad de } \\
\text { carreteras } \\
\text { (Puntaje) }\end{array}$ & $\begin{array}{c}\text { Promedio de } \\
\text { tiempo para } \\
\text { exportar e } \\
\text { importar (Días) }\end{array}$ \\
\hline INVERSIÓN & $\begin{array}{l}\text { Producto } \\
\text { interno } \\
\text { bruto[PIB] } \\
\text { (USD) }\end{array}$ & $\begin{array}{l}\text { Inversión en } \\
\text { infraestructura } \\
(\% \text { del PIB })\end{array}$ & $\begin{array}{l}\text { Participación } \\
\text { privada en } \\
\text { inversión en } \\
\text { transporte } \\
\text { (USD) }\end{array}$ & $\begin{array}{l}\text { Inversión } \\
\text { extranjera } \\
\text { directa } \\
\text { (Millones de } \\
\text { USD) }\end{array}$ \\
\hline COMPETITIVIDAD & $\begin{array}{c}\text { Índice de } \\
\text { competitividad } \\
\text { global } \\
\text { (Puntaje) }\end{array}$ & $\begin{array}{c}\text { Costo de } \\
\text { importación } \\
\text { (USD/ } \\
\text { Contenedor) }\end{array}$ & $\begin{array}{l}\text { Costo de } \\
\text { exportación } \\
\text { (USD/ } \\
\text { Contenedor) }\end{array}$ & $\begin{array}{c}\text { Costo de } \\
\text { combustible } \\
\text { (USD/Galón) }\end{array}$ \\
\hline
\end{tabular}

Elaboración propia.

Posterior a la identificación y clasificación de las variables se extraen los datos de las mismas para cada uno de los países miembros de la Alianza del Pacífico. Los datos son obtenidos de instituciones internacionales como el Banco Mundial, el Foro Económico Mundial (WEF), la Conferencia de las Naciones Unidas sobre Comercio y Desarrollo (UNCTAD), la Comisión Económica para América Latina y el Caribe (CEPAL) e instituciones y organismos de cada uno de los países. La información se representa mediante un Diagrama de Gantt, en el cual los cuatro países mencionados obtienen un puntaje o calificación en cada una de las variables relacionadas, cabe enfatizar en que el puntaje máximo que cada país puede obtener es cinco dada la normalización que se propondrá más adelante y que asimismo permitirá analizar cualesquier valor indiferente de su unidad de referencia.

Para la asignación del puntaje se debe tener en cuenta que las variables se deben clasificar dependiendo de la relación de proporcionalidad, estas pueden ser directa o inversamente proporcionales. Lo anterior se realiza para determinar si los datos analizados por país son calificados de mejor manera si éstos son mayores o menores a los de los demás países. 


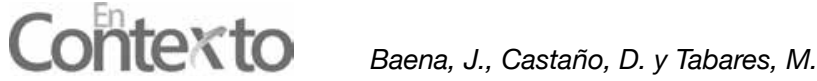

De acuerdo con lo anterior las siguientes formulas permiten e calcular la relación de proporcionalidad de las variables:

Para el caso de Relación Directamente Proporcional:

$$
X=\frac{5 \text { Puntaje } M * \text { VDMP }}{\text { Max VDMP }}
$$

Donde:

MaxVDMP (Máximo valor directamente proporcional)

5 Puntaje M (Puntaje máximo)

VDMP (Valor directamente proporcional)

Para el caso de Relación Inversamente Proporcional:

$$
\mathrm{X}=\frac{\text { MinVIMP } * 5 \text { Puntaje M }}{\text { VIMP }}
$$

Donde:

MinVIMP (Mínimo valor inversamente proporcional)

5 Puntaje M (Puntaje máximo)

VIMP (Valor inversamente proporcional)

De este modo, y gracias a las formulas aplicadas, es posible entonces realizar una sumatoria con los puntajes obtenidos en cada una de las variables evaluadas para cada país, esta sumatoria posteriormente se divide entre el número de variables. El resultado obtenido debe ser una cifra no mayor a cinco, la cual permite determinar la posición de los países en cada categoría, teniendo en cuenta que un mayor puntaje significa una mejor posición. Con la información obtenida a través de los puntajes, se tabulará y visualizará la misma información de una mejor manera, todo ello a través de gráficos radiales, gráficos de barras y demás que permitan analizar así en contexto toda la información procesada. Con estos gráficos ya realizados se procede a interpretarlos para de este modo establecer un análisis y discusión de los resultados adquiridos en el trabajo de investigación para que finalmente desde la percepción de los autores se realicen las conclusiones que sinteticen la investigación realizada. 


\section{Resultados}

\section{Infraestructura de transporte en los países de la Alianza del Pacífico}

Dentro del contexto, Sandoval (2008) define una infraestructura como un medio que permite el flujo de personas y objetos, en el que se enlazan diversos lugares, instituciones y personas en un determinado espacio social, en cada uno de estos espacios existen encargados de la disposición y orden de la infraestructura. Otros autores definen la infraestructura como un conjunto de ingeniería, instalaciones y equipos que fundamentan la prestación de los servicios dirigidos a los sectores productivos, y que puede clasificarse de acuerdo a su función y su cobertura geográfica. La infraestructura de transporte entonces se encuentra clasificada dentro de la infraestructura económica de acuerdo a la función que cumple (Perrotti y Sánchez, 2011).

Otros estudios al respecto sostienen que la infraestructura económica se considera como un recurso que favorece productividad, generación de riqueza, acceso y posicionamiento en mercados internacionales de bienes, servicios, laborales y financieros, además de ser un elemento fundamental para el desarrollo económico que proporciona escenarios de transformación (Lardé y Sánchez, 2014).
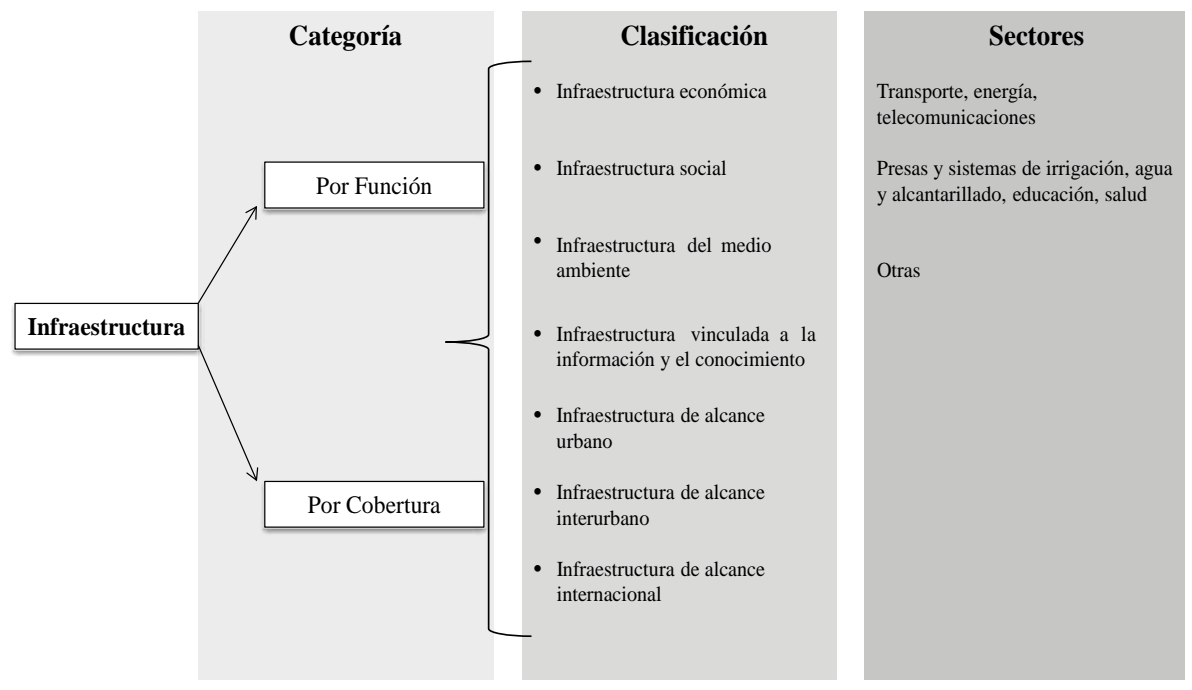

Figura 1. Clasificación de la infraestructura dentro de los Estados

Elaboración propia con datos tomados de Banco Interamericano de Desarrollo (2000). 
Por lo tanto, según la Figura 1, la infraestructura es determinante dentro del papel organizacional del Estado, de hecho otros aportes afirman que la infraestructura de transporte es la articulación de los diferentes modos de transporte y los servicios asociados de acuerdo con la geografía y los sectores económicos, su objetivo es facilitar la movilidad de carga y pasajeros entre diferentes lugares, por lo tanto se debe considerar la función de las carreteras, las vías férreas, los puertos y aeropuertos para el cumplimiento de este objetivo (Yepes, Ramírez, Villar, y Aguilar, 2013). En este sentido García (2007) establece que existe una relación entre la infraestructura de transporte y el crecimiento económico, e indica que esta relación puede ser positiva si se realiza la inversión y desarrollo adecuado de la infraestructura.

El comercio también sostiene relación con la infraestructura de transporte debido a que el acceso a mercados internacionales es proceso que involucra diversos elementos para su competitividad y productividad, los cuales generan un impacto en el territorio y en el sector privado; la adecuada infraestructura logística es uno de estos elementos, además de ser un importante elemento también es uno de los más cuestionados, pues se considera que en ocasiones esta no corresponde a los requerimientos necesarios para enfrentar el comercio internacional de manera eficaz y eficiente (Gutiérrez, 2009).

Con respecto a lo mencionado La Organización Mundial del Comercio [OMC] (2004) indica que el comercio se puede ver afectado por el estado de la infraestructura de transporte, pues si esta presenta baja calidad los costos de transporte y los tiempos de entrega se incrementan, por otro lado se ve involucrada la ventaja comparativa de aquellos sectores económicos sensibles a la calidad de la infraestructura. Por su lado Nordas y Piermartini (2004) señalan que la calidad de la infraestructura determina el desempeño comercial, pues la mejor infraestructura se asocia con mayores volúmenes comerciales, de acuerdo con sus cálculos en el caso de transporte terrestre la duplicación de los kilómetros de carreteras pavimentadas por cien kilómetros al cuadrado incrementa el comercio en un trece por ciento.

La infraestructura es además fundamental para que se dé la integración entre países, y a su vez se desarrollen actividades comerciales en el espacio económico y geográfico de una manera óptima (Rozas y Sanchéz, 2004). Perrotti y Sánchez (2011) consideran que América Latina y el Caribe como región presenta en general una brecha en infraestructura, lo cual se traduce en escasez originada por la falta de adecuación, provisión y calidad de la misma, por lo tanto se requiere articulación de las economías de la región para cerrar esta brecha. 
Las condiciones físicas generan interrupción en la conexión de la regiones, lo que convierte a la infraestructura en una prioridad en la agenda de países y regiones como en el caso que se presenta entre América del Sur y América Central, por tal razón los proyectos de infraestructura no se deben ver como un tema exclusivamente nacional (García, 2009). También los aportes de Fernández (2015) establecen que la Alianza del Pacífico es uno de estos mecanismos en América Latina que nace con el objetivo de trabajar en la integración regional con miras al posicionamiento en Asia-Pacífico. De este mecanismo son miembros Chile, Colombia, México y Perú, los cuales pretenden generar más expectativas de la integración regional que se ha visto anteriormente en Latinoamérica y trabajar en las prioridades de la región de una manera más pragmática y con mayor proyección, una de estas prioridades es la brecha presente en infraestructura, la cual limita la conexión con otros mercados.

Finalmente cabe agregar en definitiva que en América Latina y el Caribe el transporte por carretera domina la movilización de pasajeros y carga, pese a esto la infraestructura en este modo de transporte en la región presenta deficiencias vinculadas a condiciones geográficas, conectividad, calidad, altas tasas de accidentalidad y seguridad vial (Kohon, 2011).

\section{Infraestructura de transporte terrestre en Chile}

Acorde con Comisión Nacional de Investigación Científica y Tecnológica [CONICYT] (2010) el transporte terrestre es el principal modo para la movilización de mercancías en Chile a nivel nacional e internacional, las carreteras existentes conectan los nodos industriales y sus actividades productivas en las quince regiones en las que el país se encuentra dividido. Los retos que enfrenta el transporte vial en Chile se resumen en la integración de los diferentes modos de transporte, también llamado transporte intermodal y el desarrollo de sistemas de transporte inteligente y sustentable. La información anterior se complementa con las siguientes cifras obtenidas de la Dirección de Vialidad del Ministerio de Obras Publicas de Chile (2015) estas indican que el total de la red vial es de 77.801 kilómetros de los cuales el 25,14\% se encuentra pavimentado, además posee 2.587 kilómetros de longitud en dobles calzadas.

\section{Infraestructura de transporte terrestre en Colombia}

Aunque el transporte vial es la actividad con mayor participación dentro del sector de transporte, almacenamiento y comunicaciones en Colombia, su crecimiento ha desacelerado en los últimos años. El país cuenta con un rezago 
en el tema de infraestructura vial, fenómeno que se da principalmente por la baja calidad de las carreteras y por el incremento de los costos operativos de transporte de carga vial como combustibles, llantas y neumáticos, peajes, salarios, pensiones, mantenimiento y reparación (Clavijo, y otros, 2014). El rezago de la infraestructura de transporte en Colombia se da en parte por las condiciones geográficas y topográficas que limitan el desarrollo de construcción, ampliación y mantenimiento de vías (Yepes, y otros, 2013). También de acuerdo a lo anterior Jaramillo (2004) alude que un factor que incrementa los costos de transporte de las importaciones y exportaciones en Colombia es la lejanía existente entre los centros de producción y los puertos. Se agrega como dato que la red vial colombiana está compuesta por 204.855 kilómetros divididos en red vial primaria, secundaria y terciaria (Ministerio de Transporte.República de Colombia, 2015). Además Jones y Viros (2014) indican que sólo el 15\% de las carreteras del país se encuentran pavimentadas.

\section{Infraestructura de transporte terrestre en México}

El transporte terrestre es el modo más utilizado para el movimiento de carga y de pasajeros en el país. La infraestructura de transporte en México es importante porque además de que es factor de desarrollo y competitividad permite comunicar los centros de producción y consumo, eliminar desequilibrios en la región y hace más sencillo el acceso a mercados y a servicios de salud y educación. (Secretaría de Comunicaciones y Transporte, 2013). Relacionado con lo anterior la Comisión Económica para América Latina y El Caribe [CEPAL] (2012) indica el transporte terrestre presenta altos costos para operadores y usuarios, estos sobrecostos se deben especialmente al rezago de la infraestructura y al marco regulatorio que no promueve la competencia del sector. En cifras obtenidas de la Secretaría de Comunicaciones y Transporte (2013) la longitud total de la red vial mexicana es de 378.923 kilómetros de los cuales 148.329 se encuentran pavimentados.

\section{Infraestructura de transporte terrestre en Perú}

La logística y el sistema de transporte en Perú no satisfacen los requerimientos del sector productivo debido a que su oferta de servicios con valor agregado es insuficiente y los costos derivados de sus operaciones para el transporte de carga son elevados, esto se debe principalmente a la ineficiencia en las operaciones de transporte. A lo anterior se agrega que la calidad de la infraestructura de carreteras en este país se halla por debajo del promedio Latinoamericano, pues estas requieren pavimentación, conectividad entre las mismas y con los puertos, 
además del desarrollo de sistemas de gestión que articulen la tecnología y el conocimiento técnico para evitar riesgos operativos y demoras de los servicios logísticos y de transporte (Consejo Nacional de la Competitividad, 2014). De acuerdo a Provías Nacional (2014) la longitud total de la red vial el Perú es de 141.702 kilómetros de esta cifra el 14,5\% se encuentra pavimentado.

\section{Inversión y financiación de infraestructura como vector de competitividad en países y regiones}

La infraestructura es un factor determinante de la integración regional, pues el déficit en esta es catalogado como una de las principales barreras para lograr incrementar el nivel de comercio intrarregional además de la formación de un mercado regional competitivo frente al resto del mundo, por esta razón es necesario que los países pertenecientes a determinada región articulen los proyectos de inversión en infraestructura para facilitar la movilidad de bienes y personas. La cooperación resulta fundamental para el desarrollo y financiación de proyectos de infraestructura (García, 2009).

Lardé y Sánchez (2014) consideran que la inversión en proyectos de infraestructura permite aumentar la calidad y la cobertura de los servicios prestados a través de estos, pues los costos asociados a la logística y la movilidad se reducen facilitando el acceso a mercados. En el caso del transporte la inversión en proyectos de infraestructura debe estar considerada como objetivo en la agenda política de los países, debido a los impactos económicos que estos tienen en los diferentes niveles espaciales; que van desde lo urbano hasta lo internacional y en cada nivel se desarrollan determinados temas, los cuales se pueden observar en la Tabla 2. 
Tabla 2

Temas focales de la infraestructura de transporte de acuerdo al nivel espacial

\begin{tabular}{cll}
\hline $\begin{array}{c}\text { Nivel } \\
\text { espacial }\end{array}$ & \multicolumn{1}{c}{$\begin{array}{c}\text { Temas focales de la } \\
\text { infraestructura de transporte }\end{array}$} & \multicolumn{1}{c}{ Ventajas } \\
\hline Urbano & $\begin{array}{l}\text { Consecuencias de la congestión e } \\
\text { infraestructura para el transporte } \\
\text { público. }\end{array}$ & $\begin{array}{l}\text { Reducción de costos y tiempos } \\
\text { asociados a la logística. }\end{array}$ \\
\hline \multirow{2}{*}{ Regional } & $\begin{array}{l}\text { Infraestructura como un instru- } \\
\text { mento para estimular las regiones } \\
\text { menos desarrolladas. }\end{array}$ & $\begin{array}{l}\text { Cobertura del transporte } \\
\text { Conectividad regional. }\end{array}$ \\
\hline Nacional & $\begin{array}{l}\text { Equilibrio entre la inversión priva- } \\
\text { da y pública. }\end{array}$ & $\begin{array}{l}\text { Acceso a mercados } \\
\text { Crecimiento y desarrollo eco- } \\
\text { nómico. }\end{array}$ \\
\hline & $\begin{array}{l}\text { Reducción de barreras comerciales } \\
\text { Internacional aumento de la competitividad en }\end{array}$ & Posicionamiento. \\
& las economías nacionales. & \\
\hline
\end{tabular}

Elaboración propia con datos tomados de Rietveld \& Bruinsma (1998).

Considerando el impacto que tiene la inversión en las regiones Fay y Morrison (2007) indican que en la región de América Latina y el Caribe se requiere invertir más en proyectos de infraestructura para el crecimiento, competitividad y reducción de la pobreza en la región; específicamente se debe invertir entre el tres y seis por ciento del producto interno bruto (PIB), además de que la participación del Estado y el sector privado resulta fundamental, pues la articulación entre estos dos actores facilita la financiación, desarrollo, regulación y supervisión de proyectos. Considerando lo anterior otros aportes como el realizado por Serrano (2010) indican que existen diferentes alternativas para financiar proyectos de infraestructura de transporte, las cuales involucran actores del sector público y privado; caben mencionar algunas de estas como financiamiento a modo de obra pública, concesiones viales, y asociaciones público-privadas.

El financiamiento de un proyecto de infraestructura como obra pública se realiza en su totalidad con recursos del presupuesto nacional, en este caso se considera el presupuesto vigente, sus vigencias futuras y los recursos que provengan de créditos internacionales (Serrano, 2010). Por otro lado la concesión vial funciona bajo un contrato en el cual la administración pública actuando como concedente otorga a un particular o concesionario, 
la construcción y explotación de una obra de infraestructura pública durante un plazo, cabe enfatizar que el sector público conserva la propiedad de la infraestructura y no brinda ayudas ni garantías al concesionario, por lo cual este último debe asumir todos los riesgos derivados del desarrollo de sus actividades (Vassallo e Izquierdo, 2010). Igualmente Serrano (2010) manifiesta que este mecanismo de financiamiento privado desarrolla los proyectos con recursos obtenidos de los ingresos generados por los servicios prestados a los usuarios a través de la infraestructura.

Adicionalmente se destacan las alianzas público-privadas como mecanismo de financiación, estas alianzas permiten la vinculación del sector privado para suministrar bienes y servicios públicos que se asocien a una infraestructura, son mecanismos que facilitan el mantenimiento a largo plazo de los proyectos realizados bajo esta modalidad, además permiten impulsar la productividad y la eficiencia de los sectores económicos y mejorar el acceso a servicios públicos. Este mecanismo transfiere los riesgos y mecanismos de pago entre los sectores público y privado y requiere de la conformación de un contrato entre las partes (Departamento Nacional de Planeación [DNP], 2013).

Finalmente en cuanto a la financiación de infraestructura Pérez y Pastor (1998) indican que se debe considerar la participación privada en el desarrollo de proyectos de infraestructura, pues esta nace como una iniciativa ante el problema financiero del sector público; además de que es un método eficiente de desarrollar proyectos e incentivar la inversión extranjera en los mismos.

\section{Infraestructura como factor determinante de competitividad}

Porter (2008) indica que la competitividad de un país es el resultado de la productividad de las actividades económicas desarrolladas dentro del mismo y la eficiencia con la que hace uso de los recursos. La competitividad es la suma de diferentes aspectos en diferentes ámbitos, donde cada uno de estos aspectos contribuye a su mejoramiento. Si bien no existe una medida o política para establecer la competitividad, esta última debe ser el tema central en la política económica de todos los países.

Otros autores definen la competitividad como capacidad sistémica que requiere la participación y articulación de diferentes actores pertenecientes al sector público y privado para aprovechar al máximo los recursos, generar valor, incrementar el nivel de vida de la población de manera sostenible y aumentar la productividad y conocimiento; la competitividad se ha convertido en un reto para países y regiones, por tal motivo se requieren esfuerzos conjuntos que 
brinden a los mismos una posición destacada a nivel internacional (Castellanos, Ramírez, Fuquene, Quintero, y Fonseca, 2013). De acuerdo con lo anterior Sarmiento (2008) indica también que para responder a los requerimientos a nivel nacional, regional y mundial se debe conocer el comportamiento de la competitividad para así constituir políticas y estrategias que sean coherentes con los requerimientos mencionados y que se reflejen en el crecimiento continuo y en el mejoramiento de la calidad de vida de la población.

Gracias a la dinámica del contexto mundial han surgido nuevas fuentes para generar ventaja competitiva las cuales permiten trabajar con un concepto más amplio de competitividad que no simplemente se base en aspectos económicos sino que también se incluya un análisis de nuevas alternativas que pueden ser culturales, geográficas e institucionales. Uno de los temas más discutidos relacionados con la competitividad es el marco institucional, ya que este debe ser pertinente a la proyección que tenga cada país en el contexto mundial. (Baiardi y Perosa, 1999). Igualmente Sarmiento (2008) manifiesta que el concepto de competitividad se debe hacer extensivo para que obtenga la importancia que merece y se adopten los mecanismos institucionales pertinentes en los niveles nacional y regional.

Finalmente Porter (1990) considera que para generar competitividad en una nación se requiere considerar factores determinantes como: la posición que tiene el país en términos de calidad y cantidad de los factores de producción como infraestructura y mano de obra idónea, las condiciones de la demanda en el mercado interno, la existencia de empresas competitivas a nivel internacional y la estructura, estrategia y rivalidad de las empresas. Igualmente otras contribuciones agregan que la eficiencia se debe considerar también como un elemento determinante de la competitividad, teniendo en cuenta que la eficiencia se basa en el máximo aprovechamiento de los recursos (Esteban y Coll, 2003).

\section{Informe de competitividad global}

Es un informe publicado año a año desde 1979 por El Foro Económico Mundial, se define como la evaluación mundial más completa de la competitividad nacional. El informe más reciente es el desarrollado para el periodo 20152016, es este se detallan los perfiles competitivos de ciento cuarenta países y se presenta la clasificación del Índice de Competitividad Global. Este índice se encuentra basado en doce pilares que brindan una perspectiva del panorama de la competitividad en los países de todo el mundo en las diferentes etapas de desarrollo económico (World Economic Forum [WEF], 2015). 
En la Figura 2 se pueden observar los doce pilares del índice de competitividad global, estos pilares a su vez agrupan indicadores específicos que se encuentran relacionados con cada uno y facilitan su evaluación.

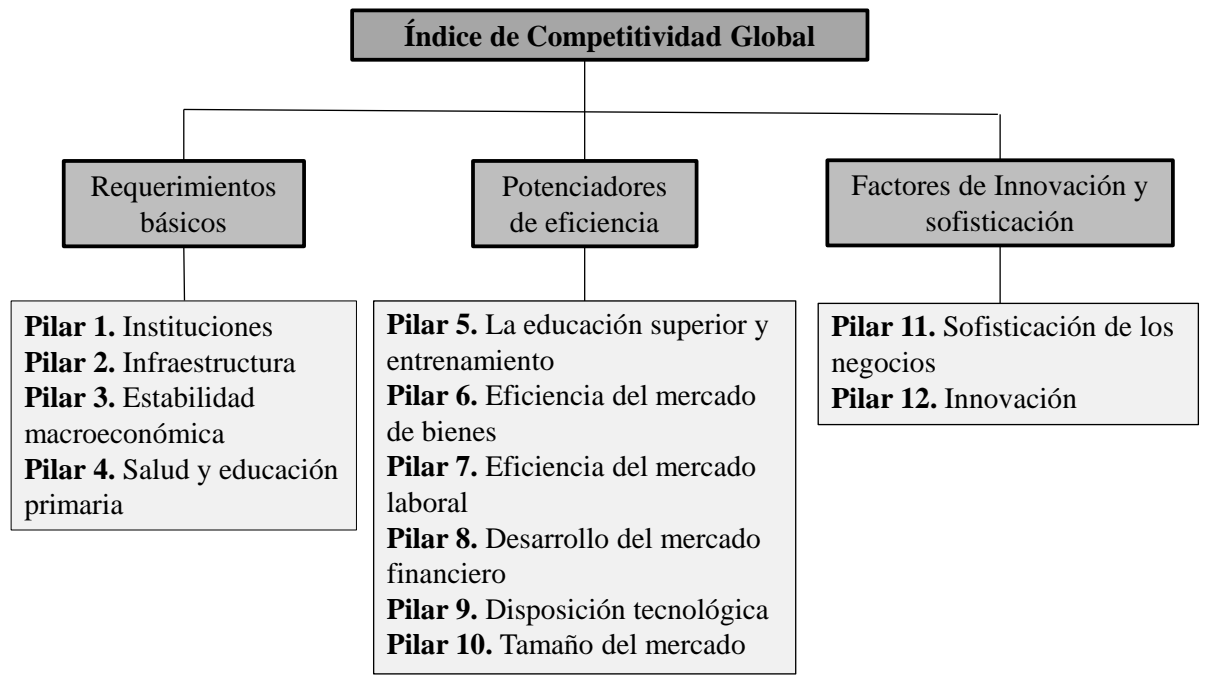

Figura 2. Pilares evaluados en Índice de Competitividad Global

Elaboración propia con datos tomados de World Economic Forum [WEF] (2015).

En el caso del pilar de infraestructura se discrimina la infraestructura de transporte, electricidad y telecomunicaciones, en infraestructura de transporte se evalúa la calidad de la infraestructura en general y específicamente la calidad de las carreteras, de la infraestructura ferroviaria, de la infraestructura portuaria y de la infraestructura de transporte aéreo (World Economic Forum [WEF], 2015).

Por lo tanto, considerando lo anterior otras contribuciones declaran que la calidad presente en las infraestructuras es protagonista en la competitividad de un país o una región frente al resto del mundo, por tal motivo se requiere la realización de un análisis sobre los niveles de gasto público e inversión y la normatividad aplicada al desarrollo de la infraestructura, para que se establezcan planes de acción que correspondan a la competitividad (Moreno y de Pablos, 1997).

\section{Análisis de información primaria}

A continuación se presentan los resultados de los tres aspectos en los cuales se enfocó la metodología. 


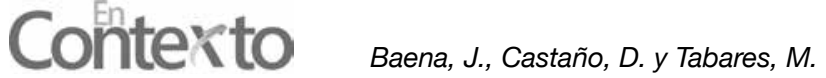

En primera instancia teniendo en cuenta la Tabla 3, es posible determinar los valores en escala de 1 a 5 , en cuyo caso 5 es el mejor valor para cada variable analizada. En este sentido aquellos países que obtienen los valores más altos son entonces aquellos que en teoría, para este caso puntual en donde se analiza la categoría sobre infraestructura de transporte terrestre, son los más preparados en términos logísticos para movilizar dentro de su espacio físico los flujos comerciales de salida y/o exportación, como también los flujos comerciales de entrada y/o importación.

Tabla 3

Variables analizadas para la primera categoría sobre infraestructura de transporte terrestre

\begin{tabular}{cccccc}
\hline País & $\begin{array}{c}\text { Densidad } \\
\text { vial }\end{array}$ & $\begin{array}{c}\text { Desempeño } \\
\text { logístico }\end{array}$ & $\begin{array}{c}\text { Calidad de } \\
\text { carreteras }\end{array}$ & $\begin{array}{c}\text { Promedio de } \\
\text { tiempo para } \\
\text { exportar e } \\
\text { importar }\end{array}$ & Total \\
\hline Chile & 2,7 & 5,0 & 5,0 & 4,3 & 4,2 \\
\hline Colombia & 4,7 & 4,0 & 2,8 & 4,3 & 3,9 \\
\hline México & 5,0 & 4,8 & 4,4 & 5,0 & 4,8 \\
\hline Perú & 2,8 & 4,4 & 3,1 & 4,0 & 3,6 \\
\hline
\end{tabular}

Elaboración propia.

Por lotanto, tras ponderar las variables, en primer lugar, densidad vial; en segundo lugar desempeño logístico, en tercer lugar, calidad de carreteras y en cuarto y último lugar, promedio de tiempo para exportar e importar. Considerando todas estas propuestas analizadas para este caso concreto es posible afirmar que México es el país con mejores condiciones de infraestructura de transporte terrestre dentro de la Alianza del Pacífico. 

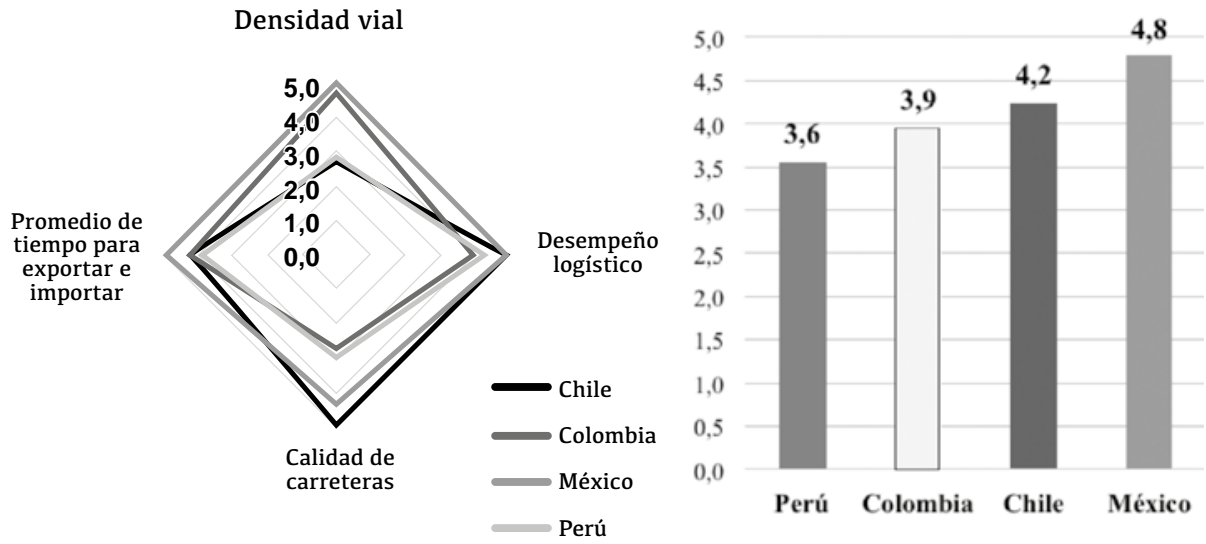

Figura 3. Análisis descriptivo de infraestructura de transporte terrestre y promedio total obtenido por país

Elaboración propia.

Cabe anotar, de acuerdo con la Figura 3 que en cada una de las variables México obtiene para la primera una puntuación de 5; para la segunda de 4,8; para la tercera de 4,4 y para la cuarta y última una puntuación de 5 lo que termina generando además en definitiva un promedio total de 4,8. De otro lado se puede notar también que Perú ocupa la última posición en la presente categoría; todo esto considerando que para la primera variable obtiene una puntuación de 2,8; para la segunda de 4,4; para la tercera de 3,1 y para la cuarta y última una puntación de 4,0; lo que conlleva a un promedio total de tan solo 3,6 en el global. Situación que asimismo sugiere que éste último país debe ser quien más debe generar esfuerzos para atender la categoría de infraestructura de transporte terrestre dentro de toda la Alianza del Pacífico, especialmente en las variables de densidad vial y calidad de carreteras cuyos valores son críticos.

En segunda instancia, y de acuerdo con la información de la Tabla 4, se pueden determinar los valores en escala de 1 a 5 , donde 5 es el mejor valor para cada variable analizada. En este sentido aquellos países que obtienen los valores más altos son aquellos que pueden desarrollar con mayor facilidad proyectos de infraestructura que permitan aumentar la calidad y la cobertura de los servicios de transporte y por ende establecer mejoras sustanciales en las condiciones de transporte terrestre, todo esto debido a que existe un mayor respaldo financiero para hacer posible la ejecución de dichos proyectos. 
Tabla 4

Variables analizadas para la segunda categoría sobre inversión

\begin{tabular}{cccccc}
\hline País & $\begin{array}{c}\text { Producto } \\
\text { interno bruto }\end{array}$ & $\begin{array}{c}\text { Inversión en } \\
\text { fraestructura } \\
\text { infraestructura }\end{array}$ & $\begin{array}{c}\text { Participación } \\
\text { privada en } \\
\text { inversión en } \\
\text { transporte }\end{array}$ & $\begin{array}{c}\text { Inversión } \\
\text { extranjera } \\
\text { directa }\end{array}$ & Total \\
\hline Chile & 1,0 & 3,2 & 0,0 & 5,0 & 2,3 \\
\hline Colombia & 1,5 & 2,7 & 4,9 & 3,5 & 3,2 \\
\hline México & 5,0 & 3,7 & 0,3 & 5,0 & 3,5 \\
\hline Perú & 0,8 & 5,0 & 5,0 & 1,7 & 3,1 \\
\hline
\end{tabular}

Elaboración propia.

Por lo tanto, al ponderar las variables, en primer lugar, producto interno bruto (PIB); en segundo lugar inversión en infraestructura, en tercer lugar, participación privada en inversión en transporte y en cuarto y último lugar, inversión extranjera directa, para este caso específico donde se contrasta la inversión es posible afirmar que México cuenta con mejores condiciones financieras para la inversión en proyectos que optimicen sus condiciones de transporte.
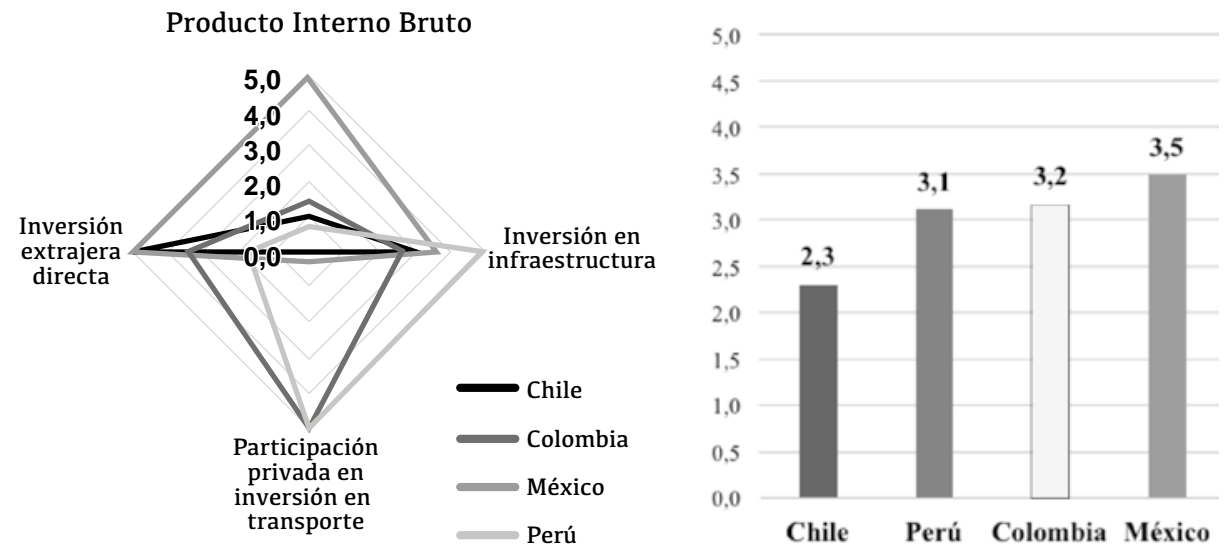

Figura 4. Análisis descriptivo de inversión y promedio total obtenido por país

Elaboración propia. 
De acuerdo con la Figura 4 se percibe que México obtiene 3,5 como mayor promedio total en la categoría de inversión, pero realizando un análisis para cada una de las variables evaluadas referentes a la categoría se puede identificar que en la primera variable evaluada obtiene un valor de 5,0; en la segunda 3,7; en la tercera 0,3 ; y en la cuarta variable obtiene 5,0; esto indica que aunque haya obtenido una mejor calificación general frente al resto de los países la variable de participación privada en inversión en transporte requiere atención ante los requerimientos de la infraestructura. Por otro lado Chile presenta el menor promedio total pues tan solo obtiene 2,3; debido a que en las primeras tres variables evaluadas consigue valores muy bajos; esto considerando que para la primera variable obtiene una puntuación de 1,0; para la segunda de 3,2; para la tercera de 0,0 ; para la cuarta y última obtiene una puntación de 5,0; pese a esto el país requiere de mayor respaldo para la financiación de proyectos que mejoren las condiciones de transporte.

Y en tercera instancia teniendo en cuenta la Tabla 5, es posible determinar los valores en escala de 1 a 5 , donde 5 es el mejor valor que se puede obtener para cada variable analizada. En este sentido aquellos países que obtienen los valores más altos son aquellos que en teoría, para este caso puntual en donde se analiza la categoría sobre competitividad, son los que han obtenido un mayor posicionamiento basado en sus condiciones de transporte como determinante de competitividad, y en consecuencia genera impactos positivos sobre el comercio internacional de mercancías y el desarrollo económico.

Tabla 5

Variables analizadas para la tercera categoría sobre competitividad

\begin{tabular}{cccccc}
\hline País & $\begin{array}{c}\text { Índice de } \\
\text { competitividad } \\
\text { global }\end{array}$ & $\begin{array}{c}\text { Costo de } \\
\text { importación }\end{array}$ & $\begin{array}{c}\text { Costo de } \\
\text { exportación }\end{array}$ & $\begin{array}{c}\text { Costo de } \\
\text { combustible }\end{array}$ & Total \\
\hline Chile & 5,0 & 5,0 & 5,0 & 3,4 & 4,6 \\
\hline Colombia & 4,7 & 1,7 & 1,7 & 4,8 & 3,2 \\
\hline México & 4,7 & 2,3 & 2,7 & 5,0 & 3,7 \\
\hline Perú & 4,6 & 4,3 & 4,6 & 3,5 & 4,3 \\
\hline
\end{tabular}

Elaboración propia. 


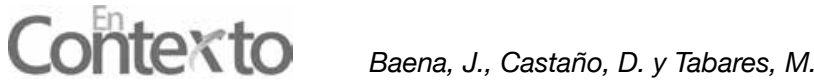

Para este caso en el cual se pretende contrastar la competitividad se ponderan las variables de índice de competitividad global, costo de importación, costo de exportación, y costo de combustible respectivamente. En la Tabla 5 se puede observar que Chile es el país más competitivo considerando las variables evaluadas para esta categoría.
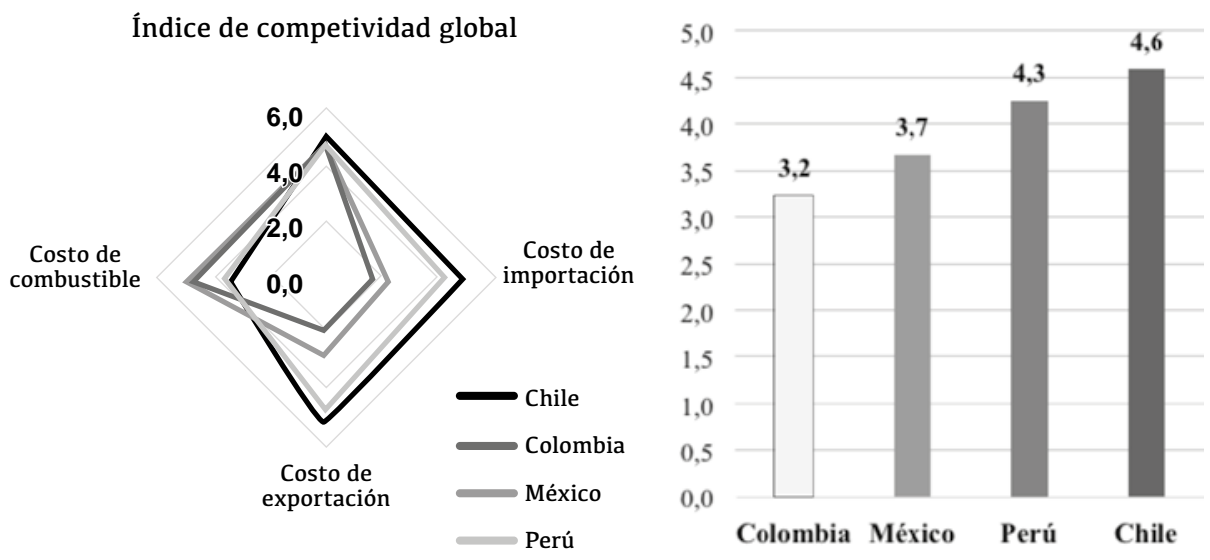

Figura 5. Análisis descriptivo de competitividad y promedio total obtenido por país

Elaboración propia.

En esta categoría es Chile quien obtiene el mayor promedio total, pues este es de 4,6 tal como se puede observar en la Figura 5, esto debido a que en la primera, segunda y tercera variable obtiene la máxima calificación; que es 5,0; en la cuarta y última variable sobre costo de combustible se manifiesta baja considerando las calificaciones obtenidas por los demás países en el marco de la Alianza del Pacífico, pues solo obtiene 3,4. De la misma manera se identifica que Colombia es el país menos competitivo en lo que respecta a sus condiciones para el transporte terrestre de carga pues en las cuatro variables evaluadas en la presente categoría obtiene una calificación de 4,7 en la primera; 1,7 en la segunda; 1,7 en la tercera y 4,8 en la cuarta y última variable, lo que conlleva a que obtenga un promedio total de 3,2 .

La anterior situación sugiere que en este último país se realice la pertinente adecuación de las condiciones de transporte terrestre para que de este modo se optimicen costos y tiempos asociados con la operación logística; lo que le permitirá entonces mejorar su posición frente a la región y frente al mundo, y de igual modo ser más competitivos en lo que respecta a comercio internacional de bienes. 


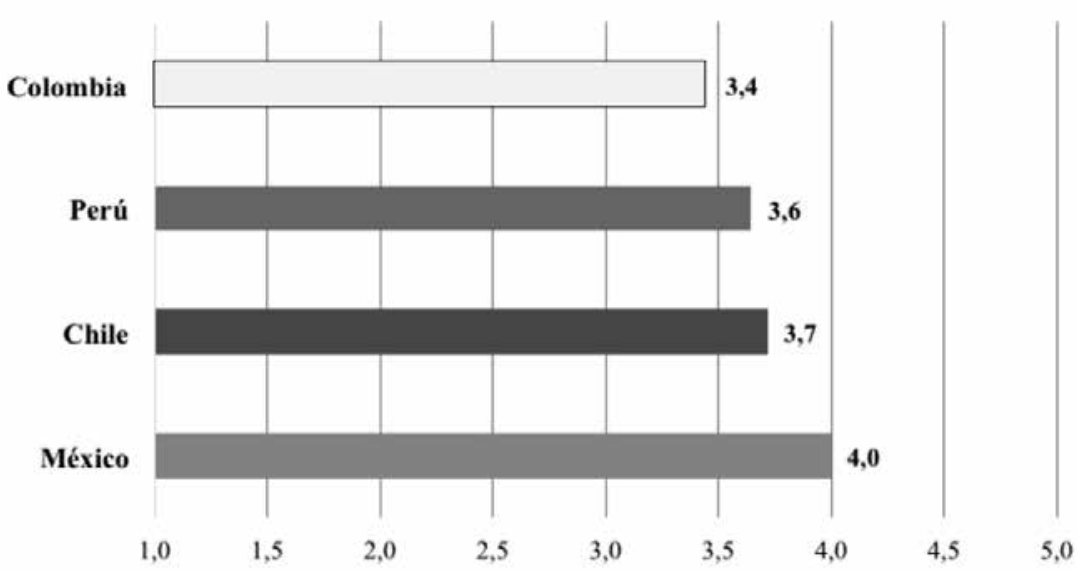

Figura 6. Promedio total obtenido de acuerdo a las tres categorías evaluadas

Elaboración propia.

En cualquier caso tras ponderar todos los valores de acuerdo a cada categoría propuesta como es el caso de infraestructura de transporte terrestre, de inversión y de competitividad; de acuerdo con la Figura 6, México se perfila entonces como el país con mayor potencial en cuanto a condiciones de transporte terrestre entre todos los demás de la Alianza del Pacífico como es evidente el caso de Chile, Perú y por último Colombia.

\section{Conclusiones}

Toda la presente investigación ha conllevado a señalar puntualmente a México, de acuerdo a toda la metodología propuesta, como el país dentro de la Alianza del Pacífico con mejores condiciones y mayor potencial en el modo de transporte terrestre seguido de Chile, Perú y Colombia. En este sentido se puede asimismo inferir que las características puntuales de la infraestructura vial de México, constituye un aspecto determinante que puede favorecer significativamente el comercio de mercancías tanto de abastecimiento como de distribución, las cuales igualmente pueden llegar a intensificarse en el tiempo con el crecimiento tras la firma de este mecanismo de integración regional.

Según el WEF Chile es el país más competitivo de todo Latinoamérica, situación que igualmente se refleja en los costos de sus exportaciones e importaciones y sirve de referente para los demás países miembros de este mecanismo de 
integración. A pesar del posicionamiento que tiene en la región debe considerar que sus condiciones para el transporte terrestre de carga deben mejorar, principalmente en lo que respecta a un aumento de su densidad vial, todo ello gracias a las inversiones que vinculen al sector público y privado.

Perú es el país dentro de este bloque comercial que mayores inversiones ha realizado para mejorar sus condiciones logísticas para el transporte de carga y así hacer frente a las barreras que aún presenta en este campo, pese a esto requiere del aumento de su red vial y de la calidad de sus carreteras para así responder a los retos de la competitividad e intensificar su participación en mercados internacionales. El caso de Colombia llama la atención considerando que en la actualidad maneja grandes volúmenes de comercio, existen evidentemente aspectos relativos a la condiciones del entorno vial que deben ser atendidos, ya que precisamente son estos los que en el análisis de los factores han influido para que esté en el último lugar detrás de Perú, Chile y México en cuanto a las condiciones de transporte en el modo vial.

Dentro del contexto la cooperación resulta ser beneficiosa y determinante para responder a los retos que presentan las regiones, esto permite a las mismas crear un ambiente propicio para el desarrollo de proyectos conjuntos entre sus países miembros que mejoren el entorno logístico en la misma e incrementen el comercio regional. De otro lado para que estos proyectos se materialicen se debe analizar el tema de la financiación, considerando que en un país y una región existen muchas prioridades para atender en beneficio de su población, por lo tanto los recursos son escasos para realizar inversiones en obras y/o proyectos para la optimización de las infraestructuras de transporte, en especial las carreteras, las cuales constituyen el eje central del presente artículo. Por tal situación se recomienda involucrar al sector privado en la financiación de obras de infraestructura vial para así desarrollarlas de una forma eficiente.

En consecuencia con todo lo ya mencionado se puede entonces deducir que las condiciones de transporte indiferente de los modos poseen relevancia en la competitividad internacional y en el contexto del comercio internacional, esto se debe a que con menores costos asociados al transporte; se logra incrementar la participación en los mercados internacionales pues los precios finales de los productos importados y exportados suelen ser más competitivos. Del mismo modo la disposición de las adecuadas condiciones físicas para el transporte por sí sola no garantiza la competitividad de este sector, pues estas deben ir 
acompañadas de una excelente gestión y de instituciones que velen por el sano desarrollo de sus operaciones.

Es importante reconocer también que si bien, cuantitativamente hablando, es difícil llevar a cabo esta medición dada la heterogeneidad en extensión, modelo de ordenamiento, topografía y demás aspectos en cada uno de los países miembros del bloque comercial; es claro también que la presente herramienta constituye una aproximación bastante llamativa que puede en efecto comparar y generar una idea global de país puede encontrarse en mejores condiciones para hacer frente a los nuevos flujos de comercio que supone la consecución y desarrollo mismo de este proyecto de integración económica regional.

Como líneas futuras de trabajo, se recomienda también implementar esta metodología para ponderar variables entre sí que no solo puedan ser numéricamente muy asimétricas, sino que puedan evidenciar proporcionalidad o razón constante entre si ya sea de naturaleza inversa o de naturaleza directa. De igual modo respecto a la presente propuesta se recomienda ahondar en otros apartados o posibles factores - aparte de la infraestructura de transporte, de la inversión y de la competitividad-, que puedan estar también relacionados con el transporte terrestre y que teóricamente puedan ser también relevantes para generar un análisis más holístico y completo en este sentido.

\section{Referencias}

Baiardi, A. y Perosa, J. (1999). Especificidades institucionais / regionais no conceito de competitividade. Organizações \& Sociedade, 6(16), 77-87.

Banco Interamericano de Desarrollo. (diciembre de 2000). Un nuevo impulso a la integración de la infraestructura regional en América del Sur. Recuperado de http://www.iirsa.org/admin_iirsa_web/Uploads/ Documents/Un\%20Nuevo\%20Impulso\%20a\%201a\%20Integracion\%20 de\%20la\%20Infraestructura.pdf

Castellanos, O., Ramírez, D., Fuquene, A., Quintero, R. y Fonseca, S. (2013). Competitividad: apropiación y mecanismos para su fortalecimiento. Bogotá, Colombia: Universidad Nacional de Colombia.

Clavijo, S., Vera, A., Malagón, A., Parga, A., Joya, S., Ortiz, M. y Ordoñez, L. (diciembre de 2014). Costos de transporte, Multimodalismo y la competitividad de Colombia. Recuperado de http://www.infraestructura. org.co/nuevapagweb/descargas/Multimodalismo.pdf 
Comisión Económica para América Latina y El Caribe [CEPAL]. (2012). Perfiles de Infraestructura y Transporte en América Latina. Caso México. Recuperado de http://www.cepal.org/perfil/noticias/noticias/7/29957/ Caso_M\%C3\%A9xico.pdf

Comisión Nacional de Investigación Científica y Tecnológica [CONICYT]. (diciembre de 2010). Investigación en transporte en Chile: Áreas de investigación y capacidades. Informe de estado del arte. Recuperado de http://www.conicyt.cl/wp-content/uploads/2012/10/articles-40714_pdf. pdf

Consejo Nacional de la Competitividad. (27 de junio de 2014). Agenda de Competitividad 2014-2018.Rumbo al Bicentenario. Recuperado de http:// www.cnc.gob.pe/images/upload/paginaweb/archivo/6/Agenda\%20 de\%20Competitividad\%202014-2018_RumboBicentenario.pdf

Departamento Nacional de Planeación [DNP]. (2013). ABECÉ Asociaciones Público Privadas. Recuperado de https://colaboracion.dnp.gov.co/CDT/ Participacin\%20privada\%20en\%20proyectos\%20de\%20infraestructu/ ABC\%20APP.pdf

Dirección de Vialidad. Ministerio de Obras Públicas.Gobierno de Chile. (agosto de 2015). Red vial nacional. Dimensionamiento y características. Recuperado de http://www.vialidad.cl/areasdevialidad/gestionvial/ Documents/Red\%20Vial\%20Nacional\%20Dimensionamiento\%20 y\%20Caracter\%C3\%ADsticas\%20A\%C3\%B1o\%202014.pdf

Esteban, J. y Coll, V. (2003). Competitividad y eficiencia. Estudios de economía aplicada, 21(3), 423-450.

Fay, M. y Morrison, M. (2007). Infrastructure in Latin America and the Caribbean. Recent Developments and Key Challenges. Recuperado de https://openknowledge.worldbank.org/bitstream/ handle/10986/7179/378990LAC0infr101OFFICIALOUSE0ONLY1. pdf?sequence $=1$

Fernández, G. (2015). La Alianza del Pacífico: Un ejemplo para la integración regional. Actualidad Jurídica Uría Menéndez, 1(39), 7-18.

García, J. (2007). ¿Existe una relación entre inversión e infraestructura de transporte y crecimiento económico? Ecos de Economía, 11(25), 1-17.

García, E. (2009). Infraestructura e Integración en América Latina. Boletín económico de ICE, Información Comercial Española, 1(2974), 27-42.

Gutiérrez,J.(2009). Infraestructura Territorial,Economíay Negocios Internacionales en Colombia. Cuadernos de Administración, 25(42), 97-108. 
Jaramillo, L. (2004). El estado de la infraestructura en Colombia frente al reto de la Globalización. Poliantea, 1(2), 129-152.

Jones, R. y Viros, C. (2014). Foundations for Growth: Infrastructure Investment in Emerging Markets. Recuperado de http://media.wix.com/ ugd/264e4c_3094ca420b4447139674a29161af2d31.pdf

Kohon, J. (2011). La Infraestructura en el Desarrollo Integral de América Latina.Diagnóstico estratégico y propuesta para una agenda prioritaria. Transporte IDeAL 2011. Recuperado de http://walk.caf.com/attach/19/ publicaciones/infraestructura/pdf/transporte.pdf

Lardé, J. y Sánchez, R. (2014). La brecha de infraestructura económica y las inversiones en América Latina. Boletin FAL.Unidad de Servicios de Infraestructura.División de Recursos Naturales e Infraestructura,CEPAL, 332(4), 1-9.

Ministerio de Transporte. República de Colombia. (19 de agosto de 2015). Transporte en Cifras. Estadísticas 2014. Recuperado de https://www. mintransporte.gov.co/

Moreno, M. y De Pablos, L. (1997). Infraestructuras del transporte terrestre y competitividad. Documentos de trabajo de la Facultad de Ciencias Económicas y Empresariales, 1(10), 1-76.

Nordas, H. y Piermartini, R. (agosto de 2004). Infrastructure and Trade. Recuperado de https://www.wto.org/english/res_e/reser_e/ ersd200404_e.doc

Organización Mundial del Comercio [OMC]. (2004). Informe sobre el comercio mundial 2004. Análisis del vínculo entre el entorno normativo nacional y el comercio internacional. Recuperado de https://www.wto.org/spanish/ res_s/booksp_s/anrep_s/world_trade_report04_s.pdf

Papí, J. (2012). Carretera e i+d+i. Carreteras: Revista técnica de la Asociación Española de la Carretera, 1(182), 190-199.

Pérez, F. y Pastor, J. (1998). La financiación privada de infraestructuras: problemas y modalidades. Perspectivas del sistema financiero, 1(62), $1-51$.

Perrotti, D. y Sánchez, R. (julio de 2011). La brecha de infraestructura en América Latina y el Caribe. Recuperado de http://idbdocs.iadb.org/ wsdocs/getdocument.aspx?docnum $=37809330$

Porter, M. (March-April de 1990). The Competitive Advantage of Nations. Recuperado de https://hbr.org/1990/03/the-competitive-advantage-of-nations 
Porter, M. (2008). ¿Qué es la competitividad? Revista de Antiguos Alumnos del IEEM, 11(14), 60-62.

Provías Nacional. (diciembre de 2014). Intervenciones en la Red Vial Nacional. Recuperado de http://www.proviasnac.gob.pe/Archivos/file/vf_RVN_ PERU_RTT_2014_(20141203).pdf

Rietveld, P. y Bruinsma, F. (1998). Is Transport Infrastructure Effective?: Transport Infrastructure and accessibility impacts on the space economy. Berlín, Heidelberg: Springer.

Rozas, P. y Sanchéz, R. (octubre de 2004). Desarrollo de infraestructura y crecimiento económico: revisión conceptual. Recuperado de http:// archivo.cepal.org/pdfs/2004/S048642.pdf

Sandoval, E. (2008). Infraestructuras transfronterizas. Un concepto para su análisis. Trayectorias, 10(26), 41-52.

Sarmiento, S. (2008). Competitividad regional. Dimensión empresarial, 6(1), 19-37.

Secretaría de Comunicaciones y Transporte. (febrero de 2013). Infraestructura de Transporte 2013-2018. Recuperado de http://www. sct.gob.mx/uploads/media/Presentacion_RMC_Infraestructura_de_ Transporte_2013-2018_01.pdf

Secretaría de Comunicaciones y Transportes. (2013). Anuario estadístico sector comunicaciones y tranportes 2013. Recuperado de http://www. sct.gob.mx/fileadmin/DireccionesGrales/DGP/estadistica/Anuarios/ Anuario-2013.pdf

Serrano, J. (2010). Financiamiento de infraestructura de transporte. Revista de Ingeniería, 1(32), 108-116.

Urrunaga Pascó-Font, R. (2009). Relevancia de la infraestructura y análisis de los sobrecostos que genera su déficit. Apuntes: Revista de Ciencias Sociales, 1(65), 61-94.

Vassallo, J. e Izquierdo, R. (2010). Infraestructura pública y participación privada: conceptos y experiencias en América y España. Recuperado de http://www.caf.com/media/3163/LibroinfraestructuraFINAL.pdf

World Economic Forum [WEF]. (2015). The Global Competitiveness Report 2015-2016. Recuperado de http://www.weforum.org/reports/globalcompetitiveness-report-2015-2016 
World Economic Forum [WEF]. (2015). The Global Competitiveness Report 2015-2016. Recuperado de http://www3.weforum.org/docs/gcr/20152016/Global_Competitiveness_Report_2015-2016.pdf

Yepes, T., Ospina, G., Aguilar, J., Calderón, L., Concha, T., Junca, J. C. y Martínez, S. (18 de diciembre de 2013). Indicadores del sector transporte en Colombia. Recuperado de http://www.repository.fedesarrollo.org.co/ bitstream/11445/173/1/Indicadores-del-sector-transporte-en-ColombiaInforme-Consolidado.pdf

Yepes, T., Ramírez, J., Villar, L. y Aguilar, J. (julio de 2013). Infraestructura de transporte en Colombia. Recuperado de http://www.repository. fedesarrollo.org.co/bitstream/11445/153/1/CDF_No_46_Julio_2013.pdf

\section{Para citar este artículo:}

Baena, J., Cataño, D. y Tabares, M. (2016). Comparativo de las condiciones de transporte terrestre de carga entre los países miembros de la Alianza del Pacífico. En-Contexto, 4(5), 155-181. 